\title{
Factors associated with adjacent-level tumor progression in patients receiving surgery followed by radiosurgery for metastatic epidural spinal cord compression
}

\author{
Vikram B. Chakravarthy, MD, ${ }^{1}$ Hammad A. Khan, BS, ${ }^{2}$ Shaarada Srivatsa, BA, ${ }^{2}$ Todd Emch, MD, ${ }^{3}$ \\ Samuel T. Chao, MD, ${ }^{4}$ and Ajit A. Krishnaney, MD1 \\ Departments of ${ }^{1}$ Neurosurgery, ${ }^{3}$ Radiology, and ${ }^{4}$ Radiation Oncology, The Cleveland Clinic; and ${ }^{2}$ Case Western Reserve \\ University School of Medicine, Cleveland, Ohio
}

\begin{abstract}
OBJECTIVE Separation surgery followed by spine stereotactic radiosurgery (SSRS) has been shown to achieve favorable rates of local tumor control and patient-reported outcomes in patients with metastatic epidural spinal cord compression (MESCC). However, rates and factors associated with adjacent-level tumor progression (ALTP) in this population have not yet been characterized. The present study aimed to identify factors associated with ALTP and examine its association with overall survival (OS) in patients receiving surgery followed by radiosurgery for MESCC.
\end{abstract}

METHODS Thirty-nine patients who underwent separation surgery followed by SSRS for MESCC were identified using a prospectively collected database and were retrospectively reviewed. Radiological measurements were collected from preoperative, postoperative, and post-SSRS MRI. Statistical analysis was conducted using the Kaplan-Meier productlimit method and Cox proportional hazards test. Subgroup analysis was conducted for patients who experienced ALTP into the epidural space (ALTP-E).

RESULTS The authors' cohort included 39 patients with a median OS of 14.7 months (range 2.07-96.3 months). ALTP was observed in 16 patients ( $41.0 \%)$ at a mean of $6.1 \pm 5.4$ months postradiosurgery, of whom 4 patients $(10.3 \%)$ experienced ALTP-E. Patients with ALTP had shorter OS (13.0 vs 17.1 months, $p=0.047)$ compared with those without ALTP. Factors associated with an increased likelihood of ALTP included the amount of bone marrow infiltrated by tumor at the index level, amount of residual epidural disease following separation surgery, and prior receipt of radiotherapy at the index level $(p<0.05)$. Subgroup analysis revealed that primary tumor type, amount of preoperative epidural disease, time elapsed between surgery and radiosurgery, and prior receipt of radiotherapy at the index level were significantly associated with ALTP-E $(p<0.05)$.

CONCLUSIONS To the authors' knowledge, this study is the first to identify possible risk factors for ALTP, and they suggest that it may be associated with shorter OS in patients receiving surgery followed by radiosurgery for MESCC. Future studies with higher power should be conducted to further characterize factors associated with ALTP in this population.

https://thejns.org/doi/abs/10.3171/2021.2.FOCUS201097

KEYWORDS metastatic epidural spinal cord compression; spine metastasis; spine stereotactic radiosurgery; adjacent-level progression; overall survival

$\mathrm{M}$ ETASTATIC epidural spinal cord compression (MESCC) is estimated to affect $5 \%-10 \%$ of all patients with cancer. ${ }^{1-3}$ If left untreated, MESCC causes severe pain, progressive neurological impairment, and eventual paraplegia with a median survival of 3-6 months. ${ }^{3,4}$ Given the debilitating nature of its complications, MESCC represents a medical emergency that requires urgent intervention.
Treatment paradigms for MESCC have rapidly shifted over the past several decades, from isolated radiotherapy or maximal resection toward a combined approach consisting of surgery followed by adjuvant radiation therapy. ${ }^{5}$ This approach has been recognized as the standard of care for MESCC since 2005, following work by Patchell et al. that demonstrated greater survival and superior rates of ambulation in patients receiving surgery followed by ra-

ABBREVIATIONS ALTP = adjacent-level tumor progression; ALTP-E = ALTP into the epidural space; ESCC $=$ epidural spinal cord compression; KPS = Karnofsky Performance Scale; $L P=$ local progression; MESCC = metastatic ESCC; OS = overall survival; SSRS = spine stereotactic radiosurgery.

SUBMITTED December 29, 2020. ACCEPTED February 16, 2021.

INCLUDE WHEN CITING DOI: 10.3171/2021.2.FOCUS201097. 
diotherapy compared with those receiving radiotherapy alone. ${ }^{6}$ More recently, the surgical aspect has evolved toward separation surgery, which involves creating a minimum 1- to 2-mm margin around the thecal sac and nerve roots to facilitate the delivery of focused radiation without compromising the spinal cord. This treatment strategy has been shown to reduce surgical morbidity. ${ }^{7-9,10}$ Spine stereotactic radiosurgery (SSRS) involves the delivery of high-dose ionizing radiation that conforms to tumor volume while minimizing radiation to surrounding intact structures. It has been shown to achieve high rates of local control and favorable patient-reported outcomes even in patients with radioresistant tumor pathologies. ${ }^{11-17}$

Separation surgery followed by SSRS has become a widely used treatment strategy for patients with MESCC. In an effort to further improve outcomes for these patients, previous studies have sought to characterize clinical and treatment-specific predictors of survival and local control following this multimodal approach to treatment. A retrospective study by Al-Omair et al. found that greater resection of epidural disease and higher doses of postoperative radiotherapy were associated with greater local control rates for patients with MESCC. ${ }^{18}$ However, rates and factors associated with adjacent-level tumor progression (ALTP) in this population have not yet been characterized, and the ability to predict and potentially circumvent ALTP is of growing significance as cancer therapies continue to improve survival for patients with spinal metastatic disease. As such, in the present study, we aimed to identify factors associated with ALTP and examine its association with overall survival (OS) in patients receiving surgery followed by radiosurgery for MESCC.

\section{Methods \\ Study Design}

This was a single-center retrospective study of patients who received separation surgery followed by SSRS for MESCC at a large academic medical center between 2009 and 2019. Patients were identified using our institution's prospectively collected spine tumor registry database following study approval by the institutional review board. Patients with a history of prior spine surgery for the same lesion, and those with primary hematological or central nervous system malignancies as determined by tumor histology were excluded from the analysis. The primary outcome measure in this study was ALTP on MRI. A subgroup analysis was conducted for patients with ALTP who experienced adjacent-level tumor progression into the epidural space (ALTP-E).

\section{Data Collection and Radiographic Measurements}

Electronic health records were retrospectively reviewed to collect patient, tumor, and treatment characteristics. Preoperative, postoperative, and post-SSRS MRI was used to measure the extent of epidural disease, bone marrow involvement, and tumor separation, as well as to ascertain local progression (LP), ALTP, ALTP-E, and the extent of epidural spinal cord compression (ESCC). All radiographic measurements were performed by an attending neuroradiologist at our institution (T.E.). Epidural disease was measured as the largest unidimensional tumor diameter. ESCC was sorted into the following categories based on Bilsky grade (cervical and thoracic lesions) or assessment of thecal sac cross-sectional area (lumbosacral lesions): no epidural compression (Bilsky grades 0, 1a), epidural compression (Bilsky grades 1b, 1c), and cord or thecal sac compression (Bilsky grades 2, 3). Marrow involvement was measured as the percentage of local vertebral body bone marrow infiltrated by tumor and was classified as $>50 \%$ abnormal or $\leq 50 \%$ abnormal. Tumor separation was defined as the distance between the tumor and spinal cord and was therefore not measured in patients with lumbosacral disease. In accordance with preliminary Spine Response Assessment in Neuro-Oncology (SPINO) group recommendations, LP was defined as any measurable unidimensional increase in epidural disease.${ }^{19} \mathrm{LP}$, ALTP, and ALTP-E were assessed using post-SSRS MRI in reference to postoperative, pre-SSRS MRI. All SSRS contours were created in accordance with consensus guidelines, using either postoperative MRI or CT myelograms if MRI was suboptimal for contouring. ${ }^{20}$ The adjacent level was included in SSRS contours only if tumor involvement was present at that level preoperatively.

\section{Statistical Analysis}

Descriptive statistics were used to examine demographic, tumor, treatment, and radiological characteristics in our cohort. Categorical variables are reported as counts and percentages, while continuous variables are reported as mean \pm standard deviation or median (range). Amount of epidural disease was analyzed as a binary categorical variable about the median value in our cohort. OS was defined as the time elapsed between surgery and death or latest follow-up visit. Time to LP, ALTP, and ALTP-E was calculated as the time elapsed between SSRS and the MRI study on which progression was first detected. Progression probabilities were determined using the Kaplan-Meier productlimit method. The Kaplan-Meier product-limit method and Cox proportional hazards test were used to examine the association of categorical and continuous variables, respectively, with OS, ALTP, and ALTP-E. All statistical analysis was conducted using SAS version 9.4 (SAS Institute Inc.). Statistical significance was defined as $\mathrm{p}<0.05$, and $\mathrm{p}<$ 0.10 was considered a trend toward statistical significance.

\section{Results}

\section{Patient and Treatment Characteristics}

Our final cohort consisted of 39 patients with a mean age of $58.3 \pm 12.8$ years and median preoperative Karnofsky Performance Scale (KPS) score of 70 (range 40-90). The most common primary tumor histology was renal cell carcinoma (14 patients, 35.9\%), followed by prostate carcinoma (7 patients, $18 \%$ ), sarcoma (5 patients, $12.8 \%$ ), and non-small cell lung carcinoma (4 patients, 10.3\%). Thirty-five patients $(89.7 \%)$ presented with high-grade ESCC (cord or thecal sac compression), and the most common primary indication for surgical intervention was pain (32 patients, $82.1 \%$ ). Twenty-nine patients $(74.4 \%)$ were treated for MESCC lesions in the thoracic spine, 8 $(20.5 \%)$ for lesions in the lumbosacral spine, and 2 (5.1\%) 
for lesions in the cervical spine. Seven patients (18\%) had received ablative radiotherapy to the target region prior to surgery. Thirty-eight patients $(97.4 \%)$ underwent separation surgery via a posterior-only approach and 1 patient via a combined anterior-posterior approach. The median number of levels decompressed was 1 (range 1-3), and the median number of levels instrumented was 4 (range $0-10$ ). SSRS was delivered at a median of 6 weeks (range 2.5718 weeks) following surgery. The median SSRS treatment dose was 16 Gy (range 15-30 Gy). Thirty-six patients (92.3\%) received high-dose single-fraction SSRS, while only 3 patients $(7.7 \%)$ received hypofractionated SSRS. These data are described in Tables 1 and 2.

\section{Radiological Measurements}

The mean extents of unidimensional local epidural disease preoperatively, postoperatively, and post-SSRS were $7.28 \pm 3.47 \mathrm{~mm}, 4.00 \pm 3.55 \mathrm{~mm}$, and $3.97 \pm 3.51 \mathrm{~mm}$, respectively. The majority of patients had $>50 \%$ local bone marrow infiltration by tumor preoperatively (23 patients, $59 \%$ ), postoperatively (23 patients, $59 \%$ ), and post-SSRS (24 patients, 61.5\%). Thirty-five patients (89.7\%) had highgrade ESCC preoperatively, 18 postoperatively (46.2\%), and 10 post-SSRS (26.3\%). These data are shown in Table 3. LP, as measured by unidimensional increase in local epidural disease, was observed in 13 patients (33.3\%) at a mean of $5.08 \pm 4.07$ months post-SSRS.

\section{Overall Survival}

The median OS in our cohort was 14.7 months (range 2.07-96.3 months). Factors significantly associated with shorter OS included ALTP ( $\mathrm{p}=0.047$, Fig. 1A), ALTP-E $(p=0.014$, Fig. 1B), receipt of ablative radiotherapy at the index level prior to surgery $(\mathrm{p}=0.012)$, and postoperative KPS score $\leq 70(p=0.001)$. Younger age was associated with shorter OS by trend $(\mathrm{p}=0.058)$ (Table 4). Patients with ALTP had a median OS of 13.0 months (range 2.2344.4 months) compared with 17.1 months (range 2.0796.3 months) for those without. Patients with ALTP-E had a median OS of 7.52 months (range 4.1-18.4 months) compared with 17.1 months (range 2.07-96.3 months) for those without. Patients who received prior radiotherapy had a median OS of 10.8 months (range 3.33-18.4 months) compared with 20.7 months (range 2.07-96.3 months) for those who did not receive prior radiotherapy. Patients with a postoperative KPS score $\leq 70$ had a median OS of 7.57 months (range 2.23-44.4 months) compared with 28.02 months (range 2.07-96.3 months) for those with a KPS score $>70$. These data are shown in Table 4 .

\section{Adjacent-Level Tumor Progression}

ALTP was observed in $16(41 \%)$ patients at a mean of $5.3 \pm 5.3$ months post-SSRS. MR images obtained in a patient with ALTP are shown in Fig. 2. ALTP was observed to occur within the SSRS treatment field in 7 of 16 (43.8\%) cases. Factors associated with ALTP included treatment with ablative radiotherapy at the index level prior to surgery ( 7 of 16 ALTP patients, $43.8 \%$ vs 0 of 23 non-ALTP patients, $0 \%$; $\mathrm{p}<0.001)$, postoperative KPS score $\leq 70(9$ of 16 patients, $56.3 \%$ vs 8 of 23 patients, $34.8 \%$; $p=0.022$ ),
TABLE 1. Patient and tumor characteristics

\begin{tabular}{|c|c|}
\hline Characteristic & Value $(n=39)$ \\
\hline \multicolumn{2}{|l|}{ Age, yrs } \\
\hline Mean (SD) & $58.31(12.86)$ \\
\hline Median (range) & $64.6(20.1-87.5)$ \\
\hline \multicolumn{2}{|l|}{ Comorbidities } \\
\hline Diabetes & $5(12.8 \%)$ \\
\hline Osteoporosis & $1(2.6 \%)$ \\
\hline Hypertension & $22(56.4 \%)$ \\
\hline Coronary artery disease & $4(10.3 \%)$ \\
\hline Other cardiac & $3(7.7 \%)$ \\
\hline Smoking & $10(25.6 \%)$ \\
\hline \multicolumn{2}{|l|}{ Tumor histology } \\
\hline Renal cell & $14(35.9 \%)$ \\
\hline Sarcoma & $5(12.8 \%)$ \\
\hline Prostate & $7(18.0 \%)$ \\
\hline Non-small cell lung & $4(10.3 \%)$ \\
\hline Other & $9(23.0 \%)$ \\
\hline Prior RT & $7(18.0 \%)$ \\
\hline \multicolumn{2}{|l|}{ Surgical treatment level } \\
\hline Cervical & $2(5.1 \%)$ \\
\hline Thoracic & $29(74.4 \%)$ \\
\hline Lumbosacral & $8(20.5 \%)$ \\
\hline \multicolumn{2}{|l|}{ Preop KPS score } \\
\hline$>70$ & $19(48.7 \%)$ \\
\hline$\leq 70$ & $20(51.3 \%)$ \\
\hline Mean score (SD) & $72.05(13.4)$ \\
\hline Median score (range) & $70(40-90)$ \\
\hline \multicolumn{2}{|l|}{ Postop KPS score } \\
\hline$>70$ & $17(43.6 \%)$ \\
\hline$\leq 70$ & $22(56.4 \%)$ \\
\hline Mean score (SD) & $73.85(11.61)$ \\
\hline Median score (range) & $80(40-90)$ \\
\hline \multicolumn{2}{|l|}{ Preop ESCC } \\
\hline No compression & 0 \\
\hline Epidural compression & $4(10.3 \%)$ \\
\hline Cord or thecal sac compression & $35(89.7 \%)$ \\
\hline \multicolumn{2}{|l|}{ Primary indication(s) for surgical procedure } \\
\hline Paresis & $18(46.2 \%)$ \\
\hline Bowel dysfunction & $2(5.1 \%)$ \\
\hline Bladder dysfunction & $3(7.7 \%)$ \\
\hline Sensory changes & $8(20.5 \%)$ \\
\hline Pain & $32(82.1 \%)$ \\
\hline Separation surgery due to radiographic criteria & $3(7.7 \%)$ \\
\hline Other & $6(15.4 \%)$ \\
\hline
\end{tabular}

$\mathrm{RT}$ = radiotherapy.

Values represent the number of patients (\%) unless stated otherwise. 
TABLE 2. Surgical and SSRS treatment characteristics

\begin{tabular}{|c|c|}
\hline Characteristic & Value \\
\hline \multicolumn{2}{|l|}{ No. of levels decompressed } \\
\hline Mean (SD) & $1.49(0.72)$ \\
\hline Median (range) & $1(1-3)$ \\
\hline 1 & $25(64.1 \%)$ \\
\hline 2 & $9(23.1 \%)$ \\
\hline 3 & $5(12.8 \%)$ \\
\hline \multicolumn{2}{|l|}{ No. of levels instrumented } \\
\hline Mean (SD) & $3.67(3.06)$ \\
\hline Median (range) & $4(0-10)$ \\
\hline 0 & $12(30.8 \%)$ \\
\hline 1 & $1(2.6 \%)$ \\
\hline 2 & $1(2.6 \%)$ \\
\hline 3 & $4(10.3 \%)$ \\
\hline 4 & $2(5.1 \%)$ \\
\hline 5 & $9(23.1 \%)$ \\
\hline 6 & $3(7.7 \%)$ \\
\hline$\geq 7$ & $7(17.9 \%)$ \\
\hline \multicolumn{2}{|l|}{ Surgical approach } \\
\hline Posterior & $38(97.4 \%)$ \\
\hline Combined anterior-posterior & $1(2.6 \%)$ \\
\hline \multicolumn{2}{|l|}{ Depth from skin to level of operation, $\mathrm{cm}$} \\
\hline Mean (SD) & $5.54(1.84)$ \\
\hline Median (range) & $5(1-9.7)$ \\
\hline \multicolumn{2}{|l|}{ Time from surgery to SSRS, wks } \\
\hline Mean (SD) & $6.77(3.18)$ \\
\hline Median (range) & $6(2.57-18)$ \\
\hline \multicolumn{2}{|l|}{ SSRS dose, cGy } \\
\hline Mean (SD) & $1761.54(373.90)$ \\
\hline Median (range) & $1600(1500-3000)$ \\
\hline \multicolumn{2}{|l|}{ SSRS treatment fractions } \\
\hline Single fraction & $36(92.3 \%)$ \\
\hline Hypofractionated & $3(7.7 \%)$ \\
\hline \multicolumn{2}{|l|}{ Reoperation } \\
\hline Same level & 5 \\
\hline Different level & 1 \\
\hline Median time to reoperation, days (range) & $25(5-1152)$ \\
\hline
\end{tabular}

Values represent the number of patients (\%) unless stated otherwise.

extent of local bone marrow involvement preoperatively (13 of 16 patients, $81.3 \%$ vs 10 of 23 patients, $43.5 \%$; $\mathrm{p}=$ 0.012 ) and postoperatively (13 of 16 patients, $81.3 \%$ vs 10 of 23 patients, 43.5\%; $\mathrm{p}=0.030$ ), and amount of epidural disease postoperatively ( 10 of 16 patients, $62.5 \%$ vs 9 of 23 patients, $39.1 \%$ with epidural disease $>3 \mathrm{~mm} ; \mathrm{p}=0.028$ ). Younger age (56.8 vs 59.4 years; $p=0.063$ ), primary tumor histology ( 5 renal cell, 4 sarcoma, 3 prostate carcinoma, 1 non-small cell lung carcinoma, 1 pheochromocytoma, 1 rectal carcinoma; $\mathrm{p}=0.098)$, and time elapsed from surgery to SSRS (7.3 vs 6.4 weeks; $p=0.096$ ) were associated with ALTP by trend.
Subgroup analysis was conducted for patients with ALTP who experienced ALTP-E. MR images obtained in a patient with ALTP-E are shown in Fig. 3. ALTP-E was observed in 4 patients (10.3\%) at a mean of $6.1 \pm 5.4$ months post-SSRS. ALTP-E was observed to occur within the SSRS treatment field in 2 of $4(50 \%)$ cases, and 2 patients with ALTP-E had oligometastatic disease while 2 had diffuse metastatic disease. Factors associated with ALTP-E were younger age (37.6 vs 60.7 years, $p=0.010$ ), primary tumor histology ( 2 sarcoma, 1 renal cell carcinoma, 1 rectal carcinoma; $p=0.017$ ), receipt of ablative radiotherapy at the index level prior to surgery ( 3 of 4 ALTP-E patients, $75 \%$ vs 4 of 35 non-ALTP-E patients, $11.4 \% ; \mathrm{p}=0.002$ ), postoperative KPS score $\leq 70$ (4 of 4 patients, $100 \%$ vs 13 of 35 patients, $37.1 \%$; $p=0.023$ ), hypofractionated radiotherapy ( 2 of 4 patients, $50 \%$ vs 1 of 35 patients, $2.9 \% ; \mathrm{p}=0.027)$, time elapsed from surgery to SSRS ( $11.2 \pm 6.29$ vs $6.3 \pm 2.28$ weeks; $p=0.013)$, and amount of epidural disease preoperatively ( 3 of 4 patients, $75 \%$ vs 16 of 35 patients, $45.7 \%$ with epidural disease $>3$ $\mathrm{mm} ; \mathrm{p}=0.035)$. These data are shown in Table 5 .

\section{Discussion}

As improved targeted oncological therapy continues to prolong life expectancy in cancer patients, the burden of spinal metastases is expected to increase. Identifying avenues that optimize management for patients with spinal metastases is therefore of increasing importance. One such avenue may involve the ability to predict and potentially circumvent ALTP in this population, as ALTP may result in symptoms similar to the original metastatic event. As such, the present study aimed to elucidate factors associated with ALTP in patients who received separation surgery followed by SSRS for MESCC and characterize its association with OS. To our knowledge, the results of this study are the first to identify clinical and treatmentspecific factors that may portend adjacent-level disease progression in this population and to suggest that it may be associated with shorter OS.

The present analysis expands on previous studies examining predictors of OS and local disease progression in patients receiving surgery followed by adjuvant radiotherapy for spinal metastases. In a retrospective review of 56 patients with MESCC, Hu et al. found that highly malignant primary tumors and the presence of visceral metastases were associated with shorter OS. ${ }^{21}$ The authors also found that receiving radiotherapy prior to surgery was associated with lower rates of local tumor control, while postoperative SSRS, when compared with imageguided intensity-modulated radiotherapy, was associated with greater rates of local control..$^{21}$ In a secondary analysis of 66 patients treated with surgery and postoperative SSRS for spinal metastases as part of phase 1-2 trials, Tao et al. identified lower KPS score as a negative prognostic factor for OS.22 However, these authors excluded patients with spinal cord compression and spinal instability, limiting comparisons to the present study. In their retrospective analysis of 80 patients with MESCC, Al-Omair et al. found that systemic chemotherapy post-SSRS was the only significant predictor of OS, while greater resection of 
TABLE 3. Radiological tumor characteristics

\begin{tabular}{cccc}
\hline \multicolumn{1}{c}{ Characteristic } & Preop & Postop & Post-SSRS \\
\hline Epidural disease, mm & & & \\
\hline Mean (SD) & $7.28(3.47)$ & $4.00(3.55)$ & $3.97(3.51)$ \\
\hline Median (range) & $7(1-23)$ & $3(0-16)$ & $2(0-11)$ \\
\hline ESCC* & & & \\
\hline No compression & 0 & $10(25.6 \%)$ & $16(42.1 \%)$ \\
\hline Epidural compression & $4(10.3 \%)$ & $11(28.2 \%)$ & $12(31.6 \%)$ \\
\hline Cord or thecal sac compression & $35(89.7 \%)$ & $18(46.2 \%)$ & $10(26.3 \%)$ \\
\hline Marrow involvement & & & \\
\hline$>50 \%$ abnormal & $23(59.0 \%)$ & $23(59.0 \%)$ & $24(61.5 \%)$ \\
\hline$\leq 50 \%$ abnormal & $16(41.0 \%)$ & $16(41.0 \%)$ & $15(38.5 \%)$ \\
\hline
\end{tabular}

Values represent the number of patients (\%) unless stated otherwise.

* One post-SSRS ESCC value is missing due to an artifact on imaging.

epidural disease and higher doses of postoperative SSRS were significant predictors of local control rates. ${ }^{18}$ Similar to the findings of Tao et al., the current analysis found that a lower postoperative KPS score was significantly associated with reduced OS. Additionally, our analysis revealed that ALTP, ALTP-E, and treatment with radiotherapy prior to surgery were associated with reduced OS within our cohort. As expected, these results suggest that patients with highly aggressive tumors and greater overall disease burden experienced shorter OS.

We found several factors that may be associated with ALTP, which was observed in a large percentage of patients in our cohort (41\%; 16 of 39 patients). The relatively high incidence of ALTP observed in our cohort may in part be attributed to the phenomenon of osseous pseudoprogression, whereby the intraosseous extent of tumor may transiently enlarge on MRI following SSRS for spinal metastases. ${ }^{23,24}$ However, osseous pseudoprogression has not previously been described as invading adjacent spinal levels, and the association between ALTP and OS in the present study indicates that ALTP may be of prognostic and therapeutic significance. Similar to previous studies of local disease progression, a history of radiotherapy at the index level prior to surgery and a lower postoperative KPS score were found to be associated with ALTP. Tumor histology and time from surgery to SSRS were associated with ALTP by trend. Further, our analysis also revealed that patients with a greater extent of local bone marrow infiltration by tumor on both preoperative and postoperative MRI were more likely to develop ALTP. Metastatic disease is known to extend into the bone marrow via Batson's vertebral plexus and lymphatic channels, and tumor infiltration of the bone marrow is typically not addressed surgically. Therefore, it can be concluded that patients with greater local bone marrow involvement are at greater risk for developing tumor extension into adjacent vertebral segments. This finding suggests that local bone marrow involvement, as noted on preoperative MRI, may serve as a potential prognostic factor for patients with MESCC. Additionally, we found that a greater amount of local epidural disease postoperatively was associated with an increased likelihood of ALTP. While reasons for this
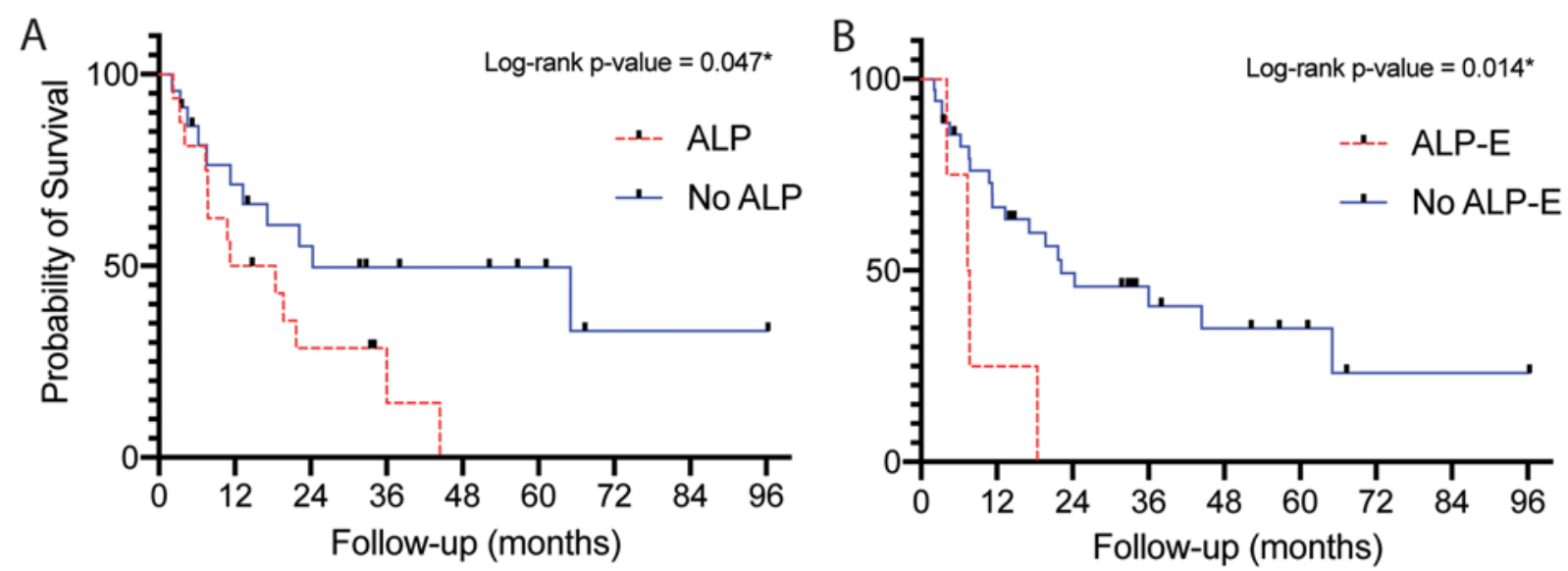

FIG. 1. A: OS for patients with ALTP (ALP; $n=16)$ versus those without ALTP $(n=23)$. B: OS for patients with ALTP-E (ALP-E; $n=$ 4) versus those without ALTP-E $(n=35)$. Asterisk denotes significance. 
TABLE 4. Predictors of OS

\begin{tabular}{ll}
\hline \multicolumn{1}{c}{ Factor } & p Value \\
\hline Age & 0.058 \\
\hline ALTP & $0.047^{*}$ \\
\hline ALTP-E & $0.014^{*}$ \\
\hline Prior RT & $0.012^{*}$ \\
\hline Postop KPS score $\leq 70$ & $0.001^{*}$ \\
\hline
\end{tabular}

* Only factors with $p<0.10$ are shown. Factors with $p<0.05$ were considered statistically significant. Factors with $p<0.10$ were considered trends toward significance.

finding are unclear, it may support a role for greater local disease resection during surgery.

Subgroup analysis of patients with ALTP-E identified similar trends, whereby patients who received prior radiotherapy at the index level and those with lower postoperative KPS scores were at greater risk of developing ALTP-E. Although ALTP-E was observed in only 4 patients (10.3\%), our analysis elucidated several additional associations that may help to better characterize patients at risk of developing it. First, patients who developed ALTP-E were more likely to have tumors with traditionally radioresistant pathologies and had received treatment with hypofractionated SSRS. Two of the patients who developed ALTP-E had oligometastatic sarcoma; one was treated with 16 Gy in 1 fraction and the other with 30 Gy across 4 fractions. Of the other 2 ALTP-E patients, 1 patient had diffusely metastatic renal cell carcinoma and was treated with 16 Gy in 1 fraction, and 1 patient had diffusely metastatic rectal carcinoma and was treated with 30 Gy across 5 fractions. While SSRS has been shown to achieve excellent rates of local control in patients with conventionally radioresistant tumors, such as sarcoma and renal cell carcinoma, the optimal SSRS dosing and fractionation for these aggressive tumors remains unclear. One recent retrospective study suggested that 24 Gy delivered in a single fraction may result in favorable rates of local control for sarcomas, while another suggested that $24 \mathrm{~Gy}$ delivered across 2 fractions may optimize outcomes for patients with renal cell carcinoma. ${ }^{25,26}$ Better delineation of these parameters may help to mitigate the increased risk of ALTP-E for patients with radioresistant tumors observed in our study. Second, our analysis revealed that increased time between separation surgery and SSRS may be associated with increased risk of ALTP-E. Since the goal of separation surgery is to achieve circumferential decompression rather than attempt gross-total resection, particularly aggressive tumors may have the opportunity to advance into the adjacent segment in the time between surgery and SSRS. Prompt delivery of adjuvant SSRS following separation surgery for MESCC may therefore help to mitigate ALTP-E. Third, patients who developed ALTP-E were more likely to have a greater amount of local epidural disease noted on preoperative MRI. Patients with greater local disease burden at the time of surgical intervention may therefore have greater risk of developing ALTP-E, possibly due to undetectable extension of tumor into the adjacent segment. This result suggests that closer surveillance and earlier intervention for spinal metastases
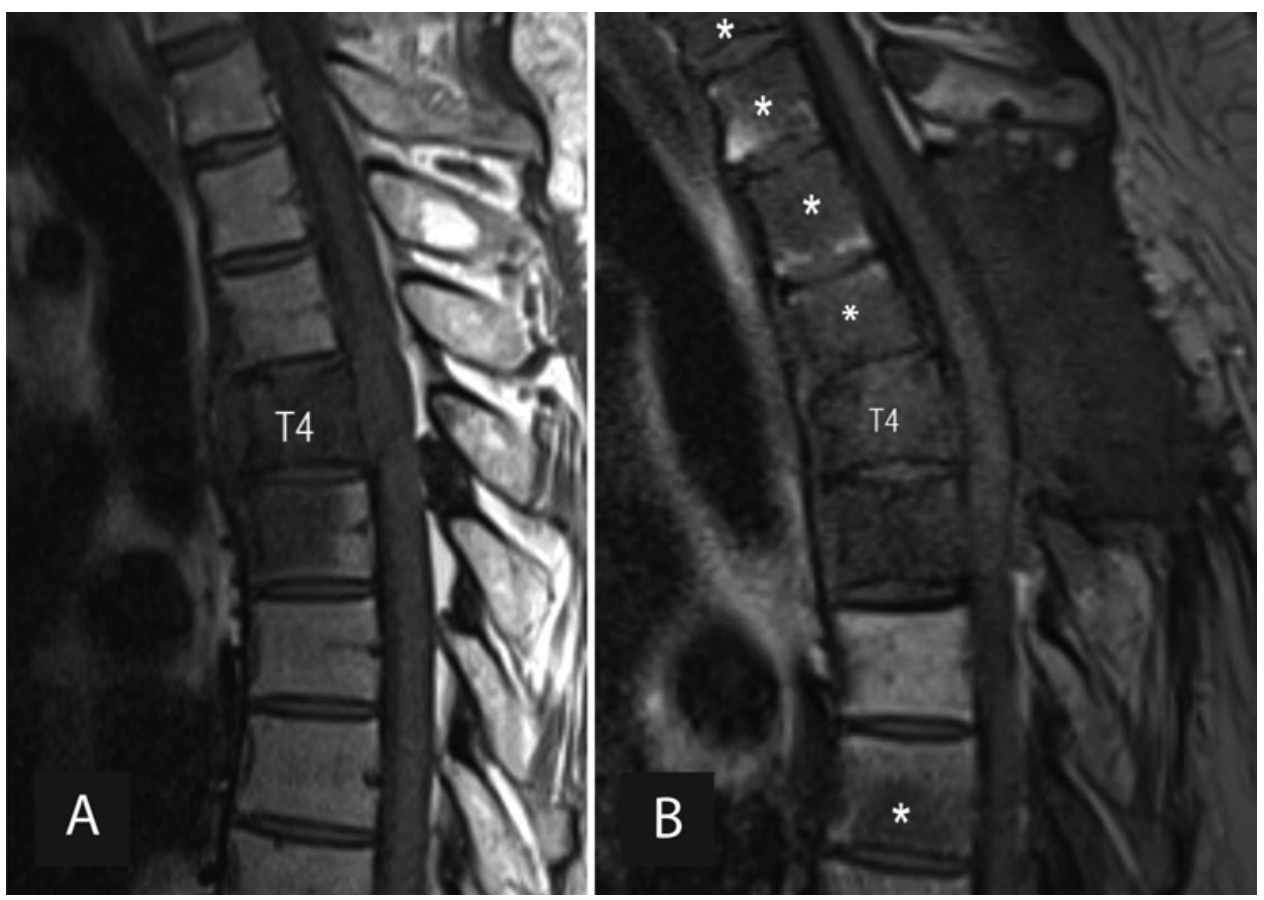

FIG. 2. Sagittal T1-weighted MR images without contrast obtained in a patient with ALTP. A: Preoperative image demonstrating metastases (low T1 signal intensity) within T4 and T5 vertebral bodies. B: Image obtained 6 months postoperatively demonstrating progressive disease at T5 and multiple new additional sites of metastases (asterisks). 

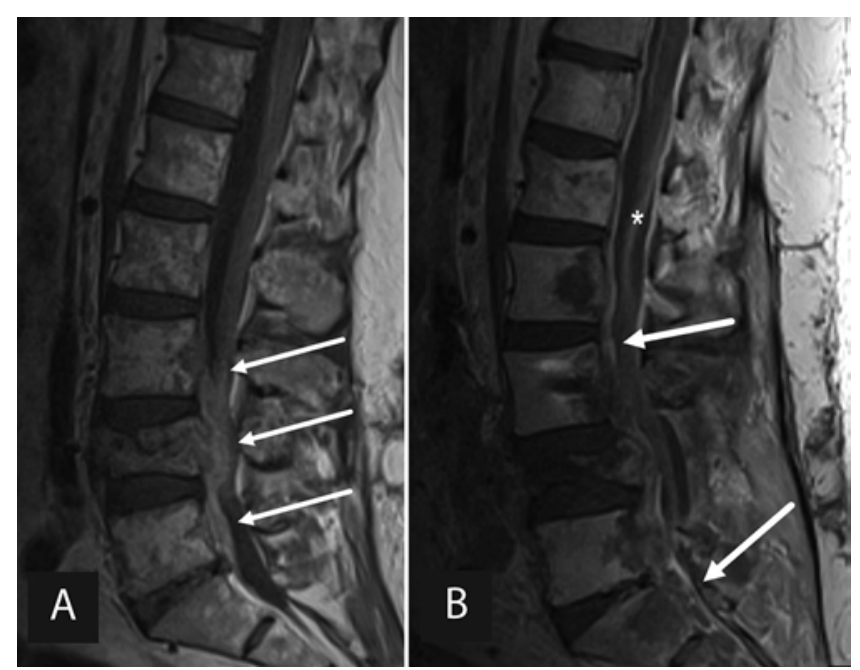

FIG. 3. Sagittal T1-weighted MR images with contrast obtained in a patient with ALTP-E. A: Preoperative image demonstrating epidural enhancement (arrows) spanning L3-5. B: Image obtained 6 months postoperatively demonstrating progression of epidural enhancement with increased cranial and caudal extent (arrows) as well as leptomeningeal disease (asterisk).

in patients with MESCC, particularly those with more aggressive known primary tumors, may be warranted.

\section{Conclusions}

Modern paradigms for the treatment of MESCC involve a multimodal approach. Large resections have been replaced by circumferential decompression of the spinal canal followed by SSRS. The primary goal of surgery is no longer to achieve complete tumor resection but rather to protect the neural elements and achieve improvements in quality of life. This study suggests a unique role for epidural disease and bone marrow involvement in the progression of spinal metastatic disease. Future studies with larger sample sizes and prospective multicenter data are needed to evaluate the effect of variable radiation dosing and fractionation schedules on ALTP. In addition, what role residual epidural disease has in osseous progression, development of compression fractures, and response to adjuvant therapies will be important clinical questions to evaluate more closely. Although current literature has encouraged surgeons to decrease aggressive surgical intervention, such intervention is perhaps warranted within the epidural space given its association with ALTP.

\section{References}

1. Bone metastasis. American Cancer Society; 2016. Accessed March 22, 2021. https://www.cancer.org/content/dam/CRC/ PDF/Public/6119.00.pdf

2. Bach F, Larsen BH, Rohde K, et al. Metastatic spinal cord compression. Occurrence, symptoms, clinical presentations and prognosis in 398 patients with spinal cord compression. Acta Neurochir (Wien). 1990;107(1-2):37-43.

3. Loblaw DA, Laperriere NJ, Mackillop WJ. A populationbased study of malignant spinal cord compression in Ontario. Clin Oncol (R Coll Radiol). 2003;15(4):211-217.

4. Rades D, Fehlauer F, Schulte R, et al. Prognostic factors for local control and survival after radiotherapy of metastatic spinal cord compression. J Clin Oncol. 2006;24(21):33883393.

5. Bilsky MH, Laufer I, Burch S. Shifting paradigms in the treatment of metastatic spine disease. Spine (Phila Pa 1976). 2009;34(22)(suppl):S101-S107.

6. Patchell RA, Tibbs PA, Regine WF, et al. Direct decompressive surgical resection in the treatment of spinal cord compression caused by metastatic cancer: a randomised trial. Lancet. 2005;366(9486):643-648.

7. Laufer I, Iorgulescu JB, Chapman T, et al. Local disease control for spinal metastases following "separation surgery" and adjuvant hypofractionated or high-dose single-fraction stereotactic radiosurgery: outcome analysis in 186 patients. $J$ Neurosurg Spine. 2013;18(3):207-214.

8. Moussazadeh N, Laufer I, Yamada Y, Bilsky MH. Separation surgery for spinal metastases: effect of spinal radiosurgery on surgical treatment goals. Cancer Contr. 2014;21(2):168-174.

9. Barzilai O, Amato MK, McLaughlin L, et al. Hybrid surgeryradiosurgery therapy for metastatic epidural spinal cord compression: a prospective evaluation using patient-reported outcomes. Neurooncol Pract. 2018;5(2):104-113.

10. Barzilai O, Boriani S, Fisher CG, et al. Essential concepts for the management of metastatic spine disease: what the surgeon should know and practice. Global Spine J. 2019;9(1) (suppl):98S-107S.

TABLE 5. Predictors of ALTP and ALTP-E

\begin{tabular}{lclll}
\hline \multicolumn{1}{c}{ Factor } & & & \multicolumn{2}{c}{ ALTP-E $(\mathrm{n}=4)$} \\
\cline { 1 - 2 } \cline { 5 - 5 } Age & $\mathrm{p}$ Value & & \multicolumn{1}{c}{ Factor } & $\mathrm{p}$ Value \\
\hline Tumor histology & 0.063 & & Age & $0.010^{*}$ \\
\hline Prior RT & 0.098 & & Tumor histology & $0.017^{*}$ \\
\hline Postop KPS score $\leq 70$ & $<0.001^{*}$ & & Prior RT & $0.002^{*}$ \\
\hline Preop marrow involvement & $0.022^{*}$ & & Postop KPS score $\leq 70$ & $0.023^{*}$ \\
\hline Postop marrow involvement & $0.012^{*}$ & & Hypofractionated RT & $0.027^{*}$ \\
\hline Postop epidural disease & $0.030^{*}$ & & Time from surgery to SSRS & $0.013^{*}$ \\
\hline Time from surgery to SSRS & $0.028^{*}$ & Preop epidural disease & $0.035^{*}$ \\
\hline
\end{tabular}

* Only factors with $p<0.10$ are shown. Factors with $p<0.05$ were considered statistically significant. Factors with $p<0.10$ were considered trends toward significance. 
11. Bilsky MH, Yamada Y, Yenice KM, et al. Intensity-modulated stereotactic radiotherapy of paraspinal tumors: a preliminary report. Neurosurgery. 2004;54(4):823-831.

12. Yamada Y, Bilsky MH, Lovelock DM, et al. High-dose, single-fraction image-guided intensity-modulated radiotherapy for metastatic spinal lesions. Int J Radiat Oncol Biol Phys. 2008;71(2):484-490.

13. Gerszten PC, Burton SA, Ozhasoglu C, Welch WC. Radiosurgery for spinal metastases: clinical experience in 500 cases from a single institution. Spine (Phila Pa 1976). 2007; 32(2):193-199.

14. Guckenberger M, Mantel F, Gerszten PC, et al. Safety and efficacy of stereotactic body radiotherapy as primary treatment for vertebral metastases: a multi-institutional analysis. Radiat Oncol. 2014;9:226.

15. Moussazadeh N, Lis E, Katsoulakis E, et al. Five-year outcomes of high-dose single-fraction spinal stereotactic radiosurgery. Int J Radiat Oncol Biol Phys. 2015;93(2):361-367.

16. Redmond KJ, Sciubba D, Khan M, et al. A Phase 2 study of post-operative stereotactic body radiation therapy (SBRT) for solid tumor spine metastases. Int J Radiat Oncol Biol Phys. 2020;106(2):261-268.

17. Bate BG, Khan NR, Kimball BY, et al. Stereotactic radiosurgery for spinal metastases with or without separation surgery. J Neurosurg Spine. 2015;22(4):409-415.

18. Al-Omair A, Masucci L, Masson-Cote L, et al. Surgical resection of epidural disease improves local control following postoperative spine stereotactic body radiotherapy. Neuro Oncol. 2013;15(10):1413-1419.

19. Thibault I, Chang EL, Sheehan J, et al. Response assessment after stereotactic body radiotherapy for spinal metastasis: a report from the SPIne response assessment in Neuro-Oncology (SPINO) group. Lancet Oncol. 2015;16(16):e595-e603.

20. Redmond KJ, Robertson S, Lo SS, et al. Consensus contouring guidelines for postoperative stereotactic body radiation therapy for metastatic solid tumor malignancies to the spine. Int J Radiat Oncol Biol Phys. 2017;97(1):64-74.

21. Hu JX, Gong YN, Jiang XD, et al. Local tumor control for metastatic epidural spinal cord compression following separation surgery with adjuvant CyberKnife stereotactic radiotherapy or image-guided intensity-modulated radiotherapy. World Neurosurg. 2020;141:e76-e85.

22. Tao R, Bishop AJ, Brownlee Z, et al. Stereotactic body radiation therapy for spinal metastases in the postoperative setting: a secondary analysis of mature phase 1-2 trials. Int $J$ Radiat Oncol Biol Phys. 2016;95(5):1405-1413.

23. Jabehdar Maralani P, Winger K, Symons S, et al. Incidence and time of onset of osseous pseudoprogression in patients with metastatic spine disease from renal cell or prostate carcinoma after treatment with stereotactic body radiation therapy. Neurosurgery. 2019;84(3):647-654.
24. Amini B, Beaman CB, Madewell JE, et al. Osseous pseudoprogression in vertebral bodies treated with stereotactic radiosurgery: a secondary analysis of prospective phase I/II clinical trials. AJNR Am J Neuroradiol. 2016;37(2):387-392.

25. Folkert MR, Bilsky MH, Tom AK, et al. Outcomes and toxicity for hypofractionated and single-fraction image-guided stereotactic radiosurgery for sarcomas metastasizing to the spine. Int J Radiat Oncol Biol Phys. 2014;88(5):1085-1091.

26. Thibault I, Al-Omair A, Masucci GL, et al. Spine stereotactic body radiotherapy for renal cell cancer spinal metastases: analysis of outcomes and risk of vertebral compression fracture. J Neurosurg Spine. 2014;21(5):711-718.

\section{Disclosures}

The authors report no conflict of interest concerning the materials or methods used in this study or the findings specified in this paper.

\section{Author Contributions}

Conception and design: Krishnaney, Chakravarthy, Khan. Acquisition of data: Chakravarthy, Khan, Srivatsa, Emch, Chao. Analysis and interpretation of data: Krishnaney, Chakravarthy, Khan, Srivatsa. Drafting the article: Chakravarthy, Khan, Srivatsa, Emch. Critically revising the article: all authors. Reviewed submitted version of manuscript: all authors. Approved the final version of the manuscript on behalf of all authors: Krishnaney. Statistical analysis: Khan. Administrative/technical/material support: Krishnaney. Study supervision: Krishnaney, Chakravarthy.

\section{Supplemental Information}

\section{Previous Presentations}

The abstract of this paper was presented virtually at the 25th Annual Scientific Meeting and Education Day of the Society for Neuro-Oncology, November 19-22, 2020.

\section{Correspondence}

Ajit A. Krishnaney: The Cleveland Clinic, Cleveland, $\mathrm{OH}$. krishna@ccf.org. 